\title{
Prognostic Factors Influencing Postoperative Development of Chronic Kidney Disease in Patients with Small Renal Tumors who Underwent Partial Nephrectomy
}

\author{
Mototsugu Muramaki Hideaki Miyake lori Sakai Masato Fujisawa
}

Division of Urology, Kobe University Graduate School of Medicine, Kobe, Japan

\author{
Key Words \\ Small renal tumor $\cdot$ Chronic kidney disease . \\ Estimated glomerular filtration rate $\cdot$ Partial nephrectomy
}

\begin{abstract}
Background: The objective of this study was to determine factors associated with the postoperative development of chronic kidney disease (CKD) following partial nephrectomy. Patients and Methods: This study included 109 patients with normal renal function treated with partial nephrectomy for small renal tumors. Of these, 73 and 36 patients underwent open partial nephrectomy (OPN) and laparoscopic partial nephrectomy (LPN), respectively. Results: Among several parameters, there was a significant difference only in the ischemia time between the OPN and LPN groups. During the median observation period of 53.4 months, CKD, defined as estimated glomerular filtration rate (eGFR) less than $60 \mathrm{ml} / \mathrm{min} / 1.73 \mathrm{~m}^{2}$, developed in 29 (39.7\%) and 14 (38.9\%) patients in the OPN and LPN groups, respectively. Univariate analysis identified age at surgery, diabetes and preoperative eGFR as significant predictors of the postoperative development of CKD; however, only age at surgery and preoperative eGFR appeared to be independently related to CKD-free survival. In fact, there was a significant difference in the CKDfree survival between patients without any independent risk factor and those with at least one of these independent risk
\end{abstract}

\section{KARGER}

Fax +4161306 1234

E-Mail karger@karger.ch

www.karger.com
(C) 2012 S. Karger AG, Basel

1015-9770/12/0063-0129\$26.00/0

Accessible online at:

www.karger.com/cur factors. Conclusions: Careful management following partial nephrectomy is necessary for elderly patients and/or those with impaired renal function, even mild, before surgery.

Copyright $\odot 2012$ S. Karger AG, Basel

\section{Introduction}

Renal cell carcinoma (RCC) is the third most common urological malignancy and accounts for approximately $3 \%$ of all cancers in adults $[1,2]$. The incidence of RCC has steadily increased over the past 3 decades, mainly due to the widespread use of imaging modalities, such as abdominal ultrasonography and computed tomography [3]. In recent years, therefore, the number of patients with small localized RCC has markedly increased, and the majority of these patients have been treated with curative surgery $[4,5]$. To date, partial nephrectomy has become a standard surgical procedure for patients diagnosed with a $\mathrm{T} 1$ tumor and a normal contralateral kidney $[6,7]$. Furthermore, the refinement of surgical techniques and instruments has led laparoscopic partial nephrectomy (LPN) to be accepted as a reliable surgical option for 
this category of patient [8]. Several contemporary studies demonstrated oncological outcomes in patients undergoing LPN equivalent to those undergoing open partial nephrectomy (OPN) $[9,10]$, however, it remains controversial whether OPN and LPN have different effects on the postoperative renal function.

In recent years, a number of studies showed a significantly increased risk of progression of renal failure, cardiovascular disease and subsequent mortality in patients developing chronic kidney disease (CKD), which is defined as having an estimated glomerular filtration rate (eGFR) less than $60 \mathrm{ml} / \mathrm{min} / 1.73 \mathrm{~m}^{2}$ [11, 12]. Accordingly, it would be potentially important in patients with small renal tumors who are treated with partial nephrectomy to prevent the development of postoperative CKD, since the majority of patients, who could be candidates for partial nephrectomy, have a normal renal function prior to surgery and will show a long disease-free survival. Considering these findings, we retrospectively reviewed the outcomes in patients with a small renal mass who underwent either OPN or LPN focusing on changes in postoperative renal function, in order to characterize the significance of possible risk factors for impairment of postoperative renal function.

\section{Patients and Methods}

Of patients who underwent either OPN or LPN at our institutions between January 2000 and December 2007, were considered for this study which included a total of 109 consecutive patients fulfilling the following criteria: a single localized unilateral renal tumor $<4.0 \mathrm{~cm}$, no suspicious finding suggesting metastatic diseases based on any imaging modalities, preoperative eGFR $\geq$ $60 \mathrm{ml} / \mathrm{min} / 1.73 \mathrm{~m}^{2}$,presence of a normal functioning contralateral kidney, and no past history of receiving any systemic therapies for renal tumors. Informed consent to perform this study was obtained from all patients, and the study design was approved by the Research Ethics Committee of our institution. All clinicopathological data were collected.

In this series, selection of the surgical procedure for resecting renal tumors (i.e., OPN or LPN) was basically determined considering the preference of the surgeon regardless of tumor characteristics, such as location and size, as well as the wishes of the patient. The surgical procedures were performed according to those previously described with minor modifications [13, 14], and irrespective of surgical procedures, renal cooling was performed on selected patients based on the judgment by the surgeon rather than the strict criterion as follows. The artery supply to the kidney was clamped and ice slush was maintained around the entire kidney for at least 5 minutes, and tumor was then resected after slush ice was minimally removed from the surgical field to maintain hypothermia in the kidney. Pathological examinations were conducted by a single pathologist according to the 2009 American
Joint Committee on Cancer TNM classification system. Generally, all patients were followed-up by laboratory and radiological evaluations as well as physical examinations every 6 months to monitor recurrence and metastasis. In the absence of a relapse of RCC 5 years after surgery, the interval between re-examinations was increased. Follow-up data on the clinicopathological characteristics of the included patients were obtained from the medical records.

To monitor the renal function, serum creatinine was measured preoperatively and at variable follow-up times in all patients. The GFR was estimated based on the modification of diet in the renal disease $\left(\right.$ MDRD) formula $\left(\mathrm{GFR}=186.3 \times \mathrm{P}^{-1.154} \times\right.$ age $^{-0.203} \times$ 0.742 [if female]) using serum creatinine and demographic variables [15]. Patients with eGFR $<60 \mathrm{ml} / \mathrm{min} / 1.73 \mathrm{~m}^{2}$ were considered to have CKD, and the time to development of CKD after surgery was defined as when eGFR initially went down to $<60$ $\mathrm{ml} / \mathrm{min} / 1.73 \mathrm{~m}^{2}$.

All statistical analyses were performed using Statview 5.0 software (Abacus Concepts, Berkeley, CA), and p values $<0.05$ were considered significant. The Chi-square test was used to analyze associations between the two groups. The CKD-free survival rates were calculated employing the Kaplan-Meier method, and differences were determined with the log-rank test. The prognostic significance of certain factors was assessed using the Cox proportional hazards regression model.

\section{Results}

Of the 109 patients included in this study, $73(67.0 \%)$ and $36(33.0 \%)$ were treated with OPN and LPN, respectively. Pathological examinations identified RCC in 94 patients $(86.2 \%)$, whereas the remaining 15 (13.8\%) were diagnosed with benign renal tumors. The characteristics of these patients are summarized in table 1 according to the surgical procedure performed. There were no significant differences in age, gender, proportion of patients with hypertension, those with diabetes, maximal tumor diameter, preoperative eGFR, frequency of renal cooling and follow-up period between OPN and LPN groups; however, ischemia time in the LPN group was significantly longer than that in the OPN group.

During the observation period of this study, CKD developed following surgery in 43 patients $(39.4 \%)$, consisting of $29(39.7 \%)$ and $14(38.9 \%)$ in OPN and LPN groups, respectively, and there was no significant difference in the incidence of postoperative CKD between these two groups. As shown in figure 1, the 1-, 3- and 5-year CKD-free survival rates of the 109 patients were 100, 90.1 and $50.6 \%$, respectively, and the median time to the development of CKD after surgery was 81.0 months. We then assessed the impact of several factors on CKD-free survival in these patients. Despite the lack of significant associations of gender, hypertension, maximal tumor di- 
Table 1. Patient characteristics according to surgical procedure

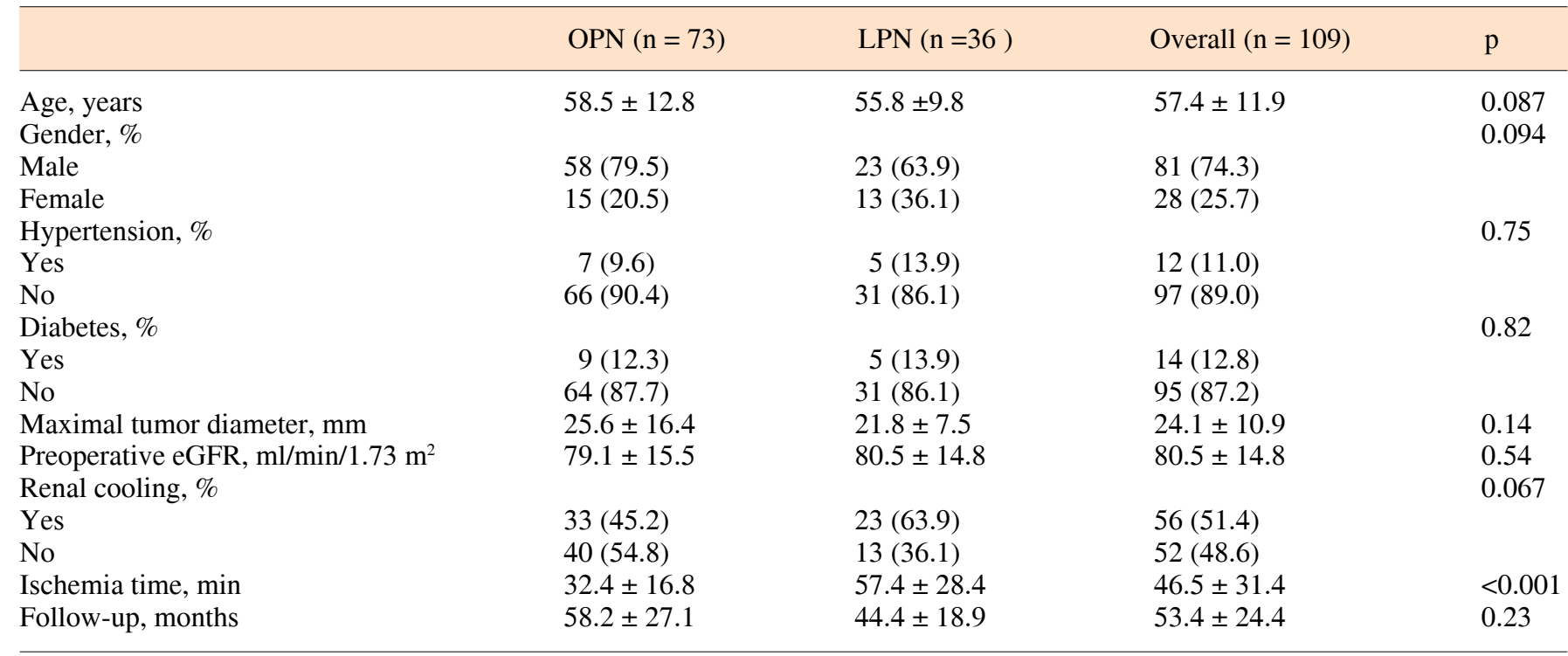

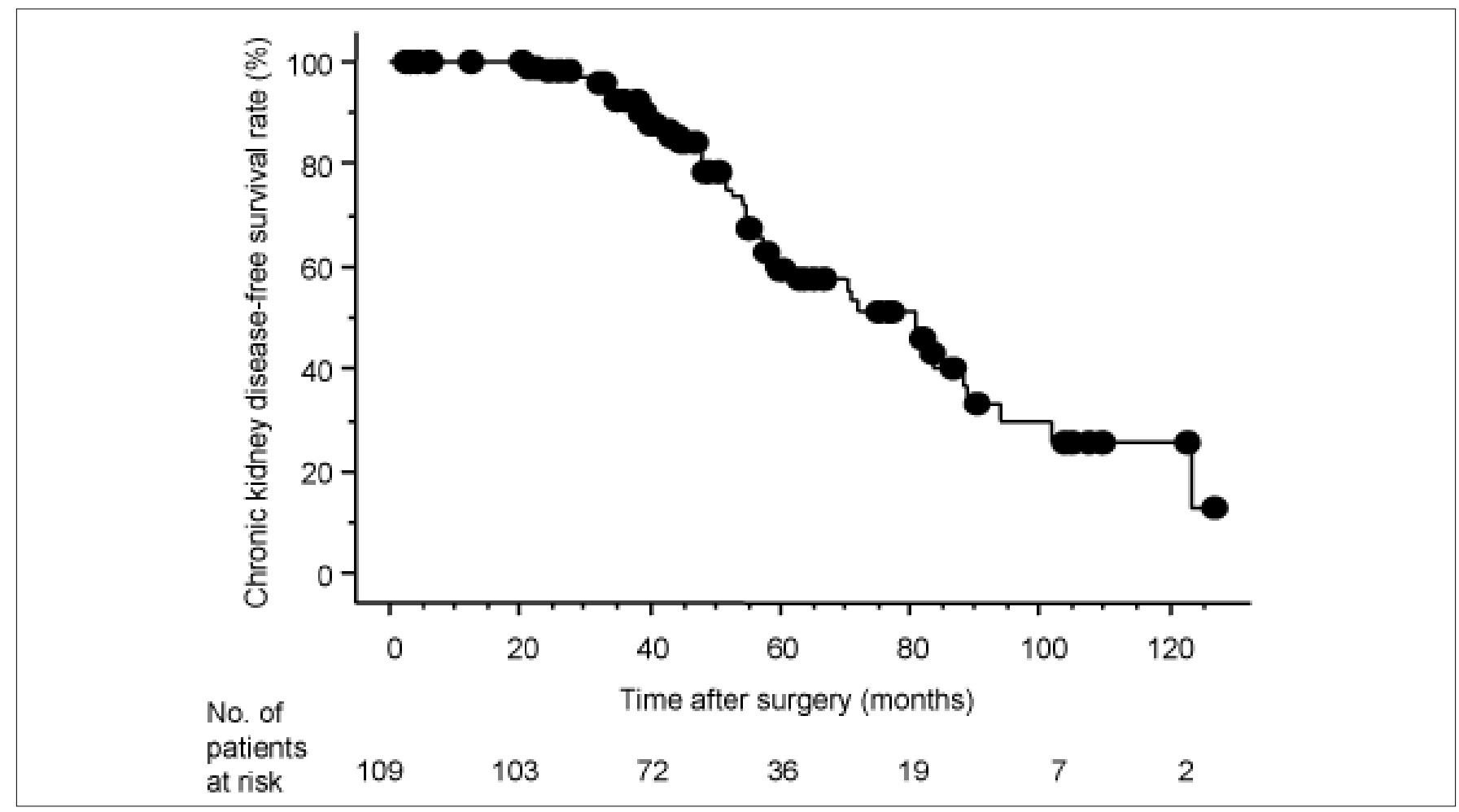

Fig. 1. CKD-free survival in patients with small renal tumors who underwent partial nephrectomy. 
ameter, renal cooling, ischemia time and surgical procedure, the age at surgery, diabetes and preoperative eGFR were identified as significant predictors of CKD-free survival on univariate analysis. Furthermore, of these 3 significant factors, age at surgery and preoperative eGFR appeared to be independently related to CKD-free survival on multivariate analysis (table 2). In fact, CKD developed in 7 of 43 patients $(16.3 \%)$ who were negative for both risk factors (i.e., 60 years $\leq$ at surgery and eGFR $\left.<70 \mathrm{ml} / \mathrm{min} / 1.73 \mathrm{~m}^{2}\right)$; however, 36 of $66(54.5 \%)$ with either single or two independent risk factors were diagnosed with CKD postoperatively. As shown in figure 2 , there was a significant difference in CKD-free survival between patients without any independent risk factor and those with at least one of these risk factors.

\section{Discussion}

Over the last decade, it was well documented that partial nephrectomy for properly selected patients with small RCC could achieve long-term cancer control similar to radical nephrectomy $[7,14,16]$. In addition, several recent studies demonstrated that, when compared to partial nephrectomy, radical nephrectomy significantly impairs the postoperative renal function, resulting in the frequent development of CKD, which has been shown to be closely associated with cardiovascular events, hospitalization and a poor health-related quality of life [12, $17,18]$. These findings strongly suggest the importance of indicating nephron sparing surgery for renal tumors when possible, and, in fact, there has been a recent trend toward a marked increase in the proportion of patients with a small renal mass who are treated with partial nephrectomy.

With the progress in the field of minimally invasive surgery, LPN has been accepted as one of the attractive options for the treatment of patients with a small renal tumor [8]. Despite several advantages of LPN, including decreased incisional morbidity, improved cosmesis and a shortened length of hospital stay, this procedure is still considered to be challenging and technically complex compared with OPN [19]. Furthermore, a limited number of studies have assessed the postoperative renal function in patients undergoing LPN compared with that in those undergoing OPN [13, 20, 21]; and therefore, there have not been any established parameters predicting the impairment of renal function following nephron sparing surgery. Considering these findings, we retrospectively analyzed the data from a total of 109 patients who un- derwent either OPN or LPN in order to identify factors associated with the onset of postoperative CKD in this cohort of patients.

We enrolled only patients with a single localized unilateral small renal tumor and normal functioning contralateral kidney in order to minimize the effects of diverse characteristics on the postoperative renal function. Of the 109 patients included in this series, 73 and 36 were treated with OPN and LPN, respectively. When their characteristics were compared according to the surgical procedure, there were no significant differences in any parameter examined, except for the ischemia time, between OPN and LPN groups. In this series, renal cooling was not applied to all patients; however, the ischemia time in patients undergoing partial nephrectomy under cold ischemia fashion appeared to be significantly longer than that in those under warm ischemia fashion (data not shown). Although there have been various studies addressing the impact of ischemia time during partial nephrectomy on the postoperative renal function, these outcomes have not been consistent [22-24]. For example, Porpigia et al. [22] reported the close association between warm ischemia time and loss of renal function estimated by renal scintigraphy, whereas Tatusgami et al. [24] found no significant differences in the postoperative total renal function between OPN and retroperitoneal LPN groups, despite the significantly longer ischemia time in the retroperitoneal LPN group than that in the OPN group. Renal artery clamping is necessary to decrease blood loss during partial nephrectomy and to allow improved visualization for performing the correct surgery. Therefore, from a theoretical point of view, every effort should be made to minimize ischemic intervals and/or protect the renal function during partial nephrectomy, such as employing a robot-associated approach, segmental renal artery clamping and cold intravascular perfusion [25-27].

During the observation period of this study, CKD developed in 43 of the 109 patients (39.4\%), and the median time to the development of CKD after surgery was 81.0 months. This outcome is equivalent or even superior to those in previous studies $[12,13,17,18]$. To explore parameters associated with the postoperative development of CKD, univariate and multivariate analyses were performed. Univariate analysis identified age at surgery, diabetes and preoperative eGFR, but not surgical procedure, as significant predictors for the onset of CKD following partial nephrectomy, of which only the age at surgery and preoperative eGFR appeared to be independently related to postoperative CKD-free survival. To date, sev- 
Table 2. Univariate and multivariate analyses of the association between various parameters and CKD-free survival

\begin{tabular}{|c|c|c|c|c|}
\hline & \multicolumn{2}{|l|}{ Univariate analysis } & \multicolumn{2}{|c|}{ Multivariate analysis } \\
\hline & HR $(95 \% \mathrm{CI})$ & $\mathrm{p}$ & $\operatorname{HR}(95 \% \mathrm{CI})$ & $\mathrm{p}$ \\
\hline Gender (male vs. female) & $1.11(0.24-3.54)$ & 0.92 & - & - \\
\hline Hypertension (yes vs. no) & $1.34(0.33-4.12)$ & 0.35 & - & - \\
\hline Diabetes (yes vs. no) & $5.82(1.34-23.33)$ & 0.034 & $1.49(0.88-7.56)$ & 0.042 \\
\hline Renal cooling (no vs. yes) & $1.13(0.78-12.56)$ & 0.15 & - & - \\
\hline Ischemia time, $\min (\geq 40$ vs. $<40)$ & $1.51(0.052-3.45)$ & 0.42 & - & - \\
\hline Surgical procedure (LPN vs. OPN) & $1.15(0.77-5.14)$ & 0.28 & - & - \\
\hline
\end{tabular}

HE = Hazard ratio.

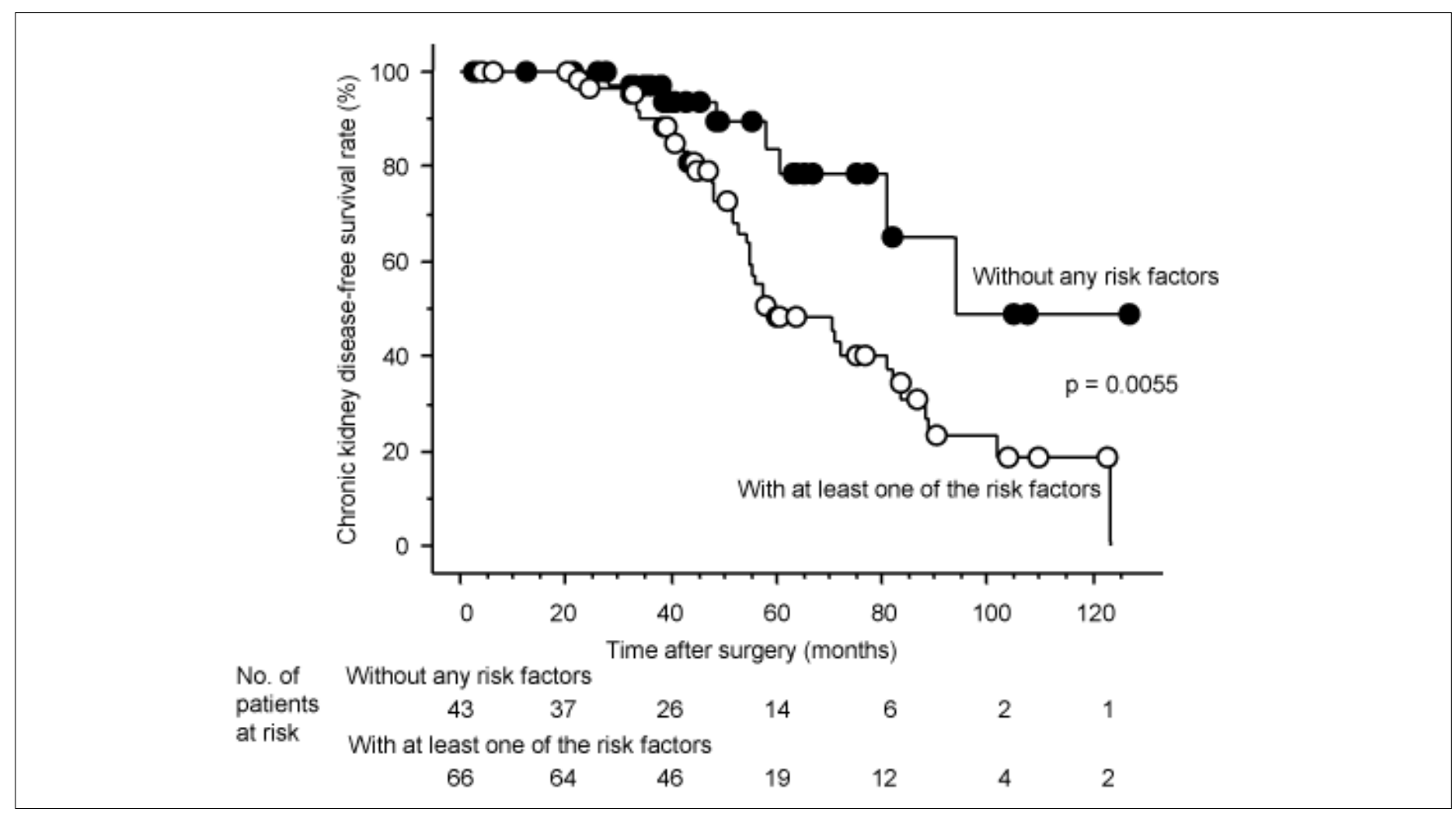

Fig. 2. Comparison of CKD-free survival in patients with small renal tumors who underwent partial nephrectomy according to the number of independent risk factors for postoperative development of CKD, including $\leq 60$ years at surgery and eGFR $<70 \mathrm{ml} / \mathrm{min} / 1.73 \mathrm{~m}^{2}$. 
eral studies have evaluated the significance of a wide variety of factors as predictors of the impairment of renal function following renal surgery [12, 13, 18, 23, 28, 29]. Although it would be difficult to interpret the outcome of these studies, which could be affected by the design of each study, such as the endpoint of renal dysfunction, the examinations for assessment of the renal function, the parameters included in statistical analysis and the application of renal cooling, age and GFR before surgery were shown to have strong effects on deterioration of the renal function after renal surgery in most of these studies $[12,13,18,28,29]$. Collectively, these findings suggest that elderly patients and/or those with a mildly impaired renal function before surgery are likely to develop CKD following partial nephrectomy irrespective of its surgical procedure. However, it should be recognized that the postoperative reduction ratio of eGFR in patients with preoperative value of eGFR $<70 \mathrm{ml} / \mathrm{min} / 1.73 \mathrm{~m}^{2}$ was significantly lower than that in those with preoperative value of eGFR $\geq 70 \mathrm{ml} / \mathrm{min} / 1.73 \mathrm{~m}^{2}$ (data not shown).

It is crucially important to develop a reliable system that enables us to exactly predict the probability of the onset of CKD after partial nephrectomy. In this series, if we divided patients into those without any independent risk factors and those with at least one independent risk factor (i.e., 60 years $\leq$ at surgery and eGFR $<70 \mathrm{ml} /$ $\min / 1.73 \mathrm{~m}^{2}$ ), there would be a significant difference in the CKD-free survival between these 2 groups. It would also be of interest to create a prediction nomogram for freedom from renal insufficiency in patients treated with partial nephrectomy. In fact, Sorbellini et al. [29] developed a nomogram that can precisely predict the 7-year probability of renal insufficiency in patients undergoing partial or radical nephrectomy by combining the following 5 parameters: age, gender, American Society of Anesthesiologists (ASA) score, preoperative serum level of creatinine and percent change in the kidney volume after surgery.

Here, we would like to emphasize several limitations of this study. First, this is a retrospective study involving a number of surgeons as operators, which could possibly affect the presented outcomes. Second, despite the comparatively longer observation period of this study compared with previous studies from high volume centers $[12,13,18,23]$, the number of patients included in this series is not large enough to draw definitive conclusions. In particular, the small number of patients treated with LPN, which has been frequently performed in recent years, may not reflect the current status of the cohort treated with partial nephrectomy. Third, there might be some other parameters more closely associated with postoperative renal function, such as change in renal parenchymal volume before and after surgery, than those examined in this study. Finally, the onset of CKD was used as the endpoint for evaluating the postoperative renal insufficiency; however, it would also be important to investigate the impact of partial nepfrectomy on other endpoints, such as the relative change in GFR following surgery, cardiovascular events and overall survival.

In conclusion, the findings of this study suggest that approximately $40 \%$ of patients who were treated with either OPN or LPN were shown to develop postoperative CKD and those elderly patients and/or those with a mildly impaired renal function prior to surgery may be likely to develop CKD following partial nephrectomy. Furthermore, the CKD-free survival in patients with at least one of these two risk factors appeared to be significantly poorer than that in those without any risk factor. Accordingly, intensive follow-up should be performed in patients undergoing partial nephrectomy considering the possible deterioration of the postoperative renal function by paying special attention to the age as well as the GFR before surgery. 


\section{References}

1 Parkin DM, Bray F, Ferlay J, Pisani P: Global cancer statistics, 2002. CA Cancer J Clin 2005;55:74-108.

-2 Jemal A, Siegel R, Ward E, Murray T, Xu J, Thun MJ: Cancer statistics, 2007. CA Cancer J Clin 2007;57:43-66.

-3 Chow WH, Devesa SS, Warren JL, Fraumeni JF Jr: Rising incidence of renal cell cancer in the United States. JAMA 1999;281:16281631.

4 Miller DC, Hollingsworth JM, Hafez KS, Daignault S, Hollenbeck BK: Partial nephrectomy for small renal masses: an emerging quality of care concern? J Urol 2006; 175:853-857.

-5 Nuttall M, Cathcart P, van der Meulen J, Gillatt D, McIntosh G, Emberton M: A description of radical nephrectomy practice and outcomes in England: 1995-2002. BJU Int 2005; 96:58-61.

6 Russo P: Open partial nephrectomy: an essential contemporary operation. Nat Clin Pract Urol 2006;3:2-3.

7 Scherr DS, Ng C, Munver R, Sosa RE, Vaughan ED Jr, Del Pizzo J: Practice patterns among urologic surgeons treating localized renal cell carcinoma in the laparoscopic age: technology versus oncology. Urology 2003; 62:1007-1011.

$>8$ Gill IS, Kavoussi LR, Lane BR, Blute ML, Babineau D, Colombo JR Jr, Frank I, Permpongkosol S, Weight CJ, Kaouk JH, Kattan MW, Novick AC: Comparison of 1,800 laparoscopic and open partial nephrectomies for single renal tumors. J Urol 2007;178:41-46.

9 Lane BR, Gill IS: 7-year oncological outcomes after laparoscopic and open partial nephrectomy. J Urol 2010;183:473-479.

10 Canes D: Long-term oncological outcomes of laparoscopic partial nephrectomy. Curr Opin Urol 2008;18:145-149.

11 Go AS, Chertow GM, Fan D, McCulloch CE, Hsu CY: Chronic kidney disease and the risks of death, cardiovascular events, and hospitalization. N Engl J Med 2004;351:1296-1305.
2 Huang WC, Levey AS, Serio AM, Snyder M, Vickers AJ, Raj GV, Scardino PT, Russo P: Chronic kidney disease after nephrectomy in patients with renal cortical tumours: a retrospective cohort study. Lancet Oncol 2006;7: 735-740.

13 Song C, Bang JK, Park HK, Ahn H: Factors influencing renal function reduction after partial nephrectomy. J Urol 2009;181:48-53.

14 Sakai I, Miyake H, Muramaki M, Kondo Y, Kusuda Y, Yamada Y, Fujisawa M: Significant impact of age at diagnosis on the prognosis of Japanese patients with pT1 renal cell carcinoma following surgical resection. BJU Int 2012;109:695-699.

15 Rocco MV, Gassman JJ, Wang SR, Kaplan RM: Cross-sectional study of quality of life and symptoms in chronic renal disease patients: the Modification of Diet in Renal Disease Study. Am J Kidney Dis 1997;29:888896.

16 Herr HW: Partial nephrectomy for unilateral renal carcinoma and a normal contralateral kidney: 10-year followup. J Urol 1999;161: 33-34.

17 Malcolm JB, Bagrodia A, Derweesh IH, Mehrazin R, Diblasio CJ, Wake RW, Wan JY, Patterson AL: Comparison of rates and risk factors for developing chronic renal insufficiency, proteinuria and metabolic acidosis after radical or partial nephrectomy. BJU Int 2009; 104:476-481.

18 Jeon HG, Jeong IG, Lee JW, Lee SE, Lee E: Prognostic factors for chronic kidney disease after curative surgery in patients with small renal tumors. Urology 2009;74:1064-1068.

19 Lane BR, Chen H, Morrow M, Anema JG, Kahnoski RJ: Increasing use of kidney sparing approaches for localized renal tumors in a community based health system: impact on renal functional outcomes. J Urol 2011;186:1229-1235.

20 Adamy A, Favaretto RL, Nogueira L, Savage C, Russo P, Coleman J, Guillonneau B, Touijer K: Recovery of renal function after open and laparoscopic partial nephrectomy. Eur Urol 2010;58:596-601.
21 Marszalek M, Meixl H, Polajnar M, Rauchenwald M, Jeschke K, Madersbacher S: Laparoscopic and open partial nephrectomy: a matched-pair comparison of 200 patients. Eur Urol 2009;55:1171-1178.

22 Porpiglia F, Fiori C, Bertolo R, Angusti T, Piccoli GB, Podio V, Russo R: The effects of warm ischaemia time on renal function after laparoscopic partial nephrectomy in patients with normal contralateral kidney. World J Urol 2012;30:257-263.

-23 Shikanov S, Lifshitz D, Chan AA, Okhunov Z, Ordonez MA, Wheat JC, Matin SF, Landman J, Wolf JS Jr, Eggener SE, Shalhav AL: Impact of ischemia on renal function after laparoscopic partial nephrectomy: a multicenter study. J Urol 2010;183:1714-1718.

-24 Tatsugami K, Eto M, Yokomizo A, Kuroiwa K, Inokuchi J, Tada Y, Takeuchi A, Seki N, Naito S: Impact of cold and warm ischemia on postoperative recovery of affected renal function after partial nephrectomy. J Endourol 2011;25:869-873.

-25 Rogers C, Sukumar S, Gill IS: Robotic partial nephrectomy: the real benefit. Curr Opin Urol 2011;21:60-64.

-26 Marley CS, Siegrist T, Kurta J, O’Brien F, Bernstein M, Solomon S, Coleman JA: Cold intravascular organ perfusion for renal hypothermia during laparoscopic partial nephrectomy. J Urol 2011;185:2191-2195.

27 Shao P, Qin C, Yin C, Meng X, Ju X, Li J, Lv Q, Zhang W, Xu Z: Laparoscopic partial nephrectomy with segmental renal artery clamping: technique and clinical outcomes. Eur Urol 2011;59:849-855.

28 Godoy G, Ramanathan V, Kanofsky JA, O'Malley RL, Tareen BU, Taneja SS, StifeIman MD: Effect of warm ischemia time during laparoscopic partial nephrectomy on early postoperative glomerular filtration rate. J Urol 2009;181:2438-2443.

29 Sorbellini M, Kattan MW, Snyder ME, Hakimi AA, Sarasohn DM, Russo P: Prognostic nomogram for renal insufficiency after radical or partial nephrectomy. J Urol 2006;176:472-476. 